\section{NEURODEGENERATIVE DISORDERS}

\section{Reversing the impairment}

The loss of short-term memory that is one of the most debilitating symptoms of Alzheimer's disease (AD) results partly from disrupted neuronal function in the entorhinal cortex, which projects to the hippocampus. In the $\mathrm{AD}$ brain these two areas have reduced levels of brainderived neurotrophic factor (BDNF), a protein that has multiple effects on synaptic plasticity and is important for learning and memory. Tuszynski and colleagues tested the effect of therapeutic gene delivery of $B D N F$ to the entorhinal cortex in several models of AD.

The primary culprit in the pathophysiology of $\mathrm{AD}$ is thought to be amyloid- $\beta(A \beta)$, the product of the amyloid precursor protein gene $(\underline{A P P})$. Transgenic mice that express a form of human $A P P$ containing two mutations linked to early-onset familial AD exhibit the main features of $\mathrm{AD}$, including cortical plaques, cell loss in the entorhinal cortex and cognitive decline by 6-7 months. The authors injected a lentiviral vector expressing BDNF-green fluorescent protein (GFP) or GFP alone bilaterally into the entorhinal cortex of these mice at age 6 months. One month later, the transgenic mice that received $g f p$ alone ( $\mathrm{TG}^{\mathrm{GFP}}$ mice) showed impaired spatial memory in the Morris water maze, whereas those that received $B D N F-g f p$ (TG ${ }^{\mathrm{BDNF}}$ mice) performed as well as wild-type (WT) control mice. The $\mathrm{TG}^{\mathrm{BDNF}}$ mice also outperformed the $\mathrm{TG}^{\mathrm{GFP}}$ mice on a test of hippocampus-dependent fear conditioning.
Immunolabelling tests confirmed high levels of BDNF expression in the entorhinal cortex of the $\mathrm{TG}^{\mathrm{BDNF}}$ mice and showed that the BDNF had also been trafficked into the hippocampus. Further investigations showed rescued expression of synaptic markers in these areas in the $\mathrm{TG}^{\mathrm{BDNF}}$ mice, as well as enhanced phosphorylation of extracellular-signal-regulated kinase (ERK) (indicative of improved cell signalling) and partial restoration of WT patterns of gene expression.

Neuron number and amyloid plaque density were not affected, so the authors concluded that BDNF may exert its effects without directly modulating $A \beta$.

The authors also tested the effects of BDNF delivery on cognitive impairment resulting from normal aging. Four-week infusion of recombinant $\mathrm{BDNF}$ into the medial entorhinal cortex of cognitively impaired aged rats again improved spatial learning and memory performance and restored levels of ERK phosphorylation, and BDNF injection into this region again partially restored gene expression. Similarly, in aged monkeys with impaired visuospatial learning, bilateral injection of vectors expressing BDNF targeted at entorhinal cortex neurons that project to the hippocampus dramatically improved performance on a visuospatial discrimination task.

In an in vitro model of neuron death, BDNF treatment prevented entorhinal cortex neurons succumbing to exposure to the toxic A $\beta$ peptide. BDNF had similar neuroprotective effects in vivo: in rats and monkeys that underwent perforant path transection and lesioning, respectively, injection of lentiviral vectors expressing BDNF into the entorhinal cortex at least partially protected this region from the substantial neuron loss that was sustained by control animals.

This study shows that therapeutic delivery of BDNF to the entorhinal cortex can ameliorate many of the hallmarks of $\mathrm{AD}$ in various models, apparently through $\mathrm{A} \beta$-independent mechanisms. The intraparenchymal infusion and gene delivery techniques used have already been tested in clinical trials, so these results hold promise for new treatments for $\mathrm{AD}$ that might complement treatments targeting the $\mathrm{A} \beta$ peptide.

Craig Nicholson

ORIGINAL RESEARCH PAPER Nagahara, A. H. et al. Neuroprotective effects of brain-derived neurotrophic factor in rodent and primate models of Alzheimer's disease. Nature Med. 8 Feb 2009 (doi:10.1038/nm.1912)

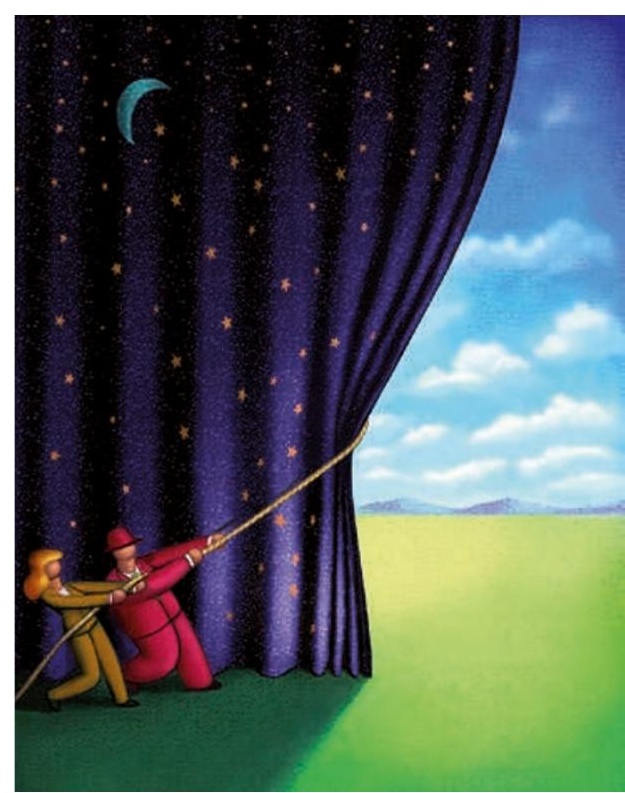

